\title{
A Pork Butcher Threatened by Pork Disease: Experience of Lomé Teaching Hospital
}

\author{
Komi Assogba ${ }^{1 *}$, Kossivi M Apetse ${ }^{1}$, Majeste Ihou-Wateba ${ }^{2}$, Mounerou Salou ${ }^{3}$, Hamza D Sama ${ }^{4}$, Komi I \\ Agbotsou $^{1}$, Edem K Mossi ${ }^{5}$, Vinyo A Codjia ${ }^{1}$, Abdoullah Blakime ${ }^{1}$, Damelan Kombate ${ }^{1}$ and Koffi AA Balogou ${ }^{1}$
}

${ }^{1}$ Department Neurology, University of Lome, Togo

${ }^{2}$ Department of Infectious Disease, University of Lome, Togo

${ }^{3}$ Laboratory of molecular biology, University of Lome, Togo

${ }^{4}$ Department of intensive care and resuscitation, University of Lome, Togo

${ }^{5}$ Department of Internal Medicine, University of Lome, Togo

*Corresponding author: Assogba K, Professor of Neurology, Campus teaching hospital of Lomé, PO Box: 30284,Togo.

Received Date: September 28, 2018

Published Date: October 16, 2018

\section{Abstract}

Introduction: Streptococcus suis meningitis, a pathogen zoonotic of pigs, is a rare infection in human. S. suis infection is acquired by handling or eating undercooked pork products. The objective was to report a rare case of infection related to occupational close contact with pork products.

Case presentation: A 32-year-old pigs farmer was admitted for severe headaches and intense agitation starting 72 hours ago. At the second day of admission, he felt cold with fever $38.5^{\circ} \mathrm{C}$, agitation, with severe headaches and vomiting. The neck became stiff with positive Kernig and Brudzinski signs. The second CSF was turbid. while waiting for the ADST and the CSF result empirical antibiotics were administrated.

Laboratory findings : Laboratory results had shown increased leukocyte count, increased of protein and glucose concentration level and no organism on Gram stain. The diagnosis of bacterial meningitis was made according to clinical and laboratory signs. CSF culture grew microorganisms which were Gram-positive cocci either in chains or in pairs were catalase- negative. They were identified as Streptococcus suis serotype I. The sample was sent to another lab in France which confirmed three weeks later the same serotype. The microbacteria were sensitive to penicillin and levofloxacin and resistant to clindamycine and tetracycline. After fifteen days of treatment and follow up, all the meningeal symptoms were stopped, and the patient had good recovery with deaf or hearing distrubance.

Conclusion: Most of the time meningitis patients are often admitted to psychiatry because of agitation symptoms. Surveillance and good clinical exam help to adjust exact diagnostic with appropriate treatment leading to better outcome. The $S$. suis infection must be born in mind in the context and history of pig's contact.

Key words: Meningeal syndrome; Bacterial meningitis; Streptococcus suis

\section{Introduction}

Streptococcus suis (S. suis) is a neglected zoonotic pathogen found primarily in pigs, that can cause serious infection in humans [1-3]. These bacterial infections have raised serious concerns in public hygien and economic field. This zoonotic pathogen has been identified as the most common and the third leading cause of bacterial meningitis in adults in South-East Asia, and northern Europe [4,5]. Due to high pork consumption, S. suis infection is endemic worldwide [3,5]. However, most clinicians and microbiologists are unfamiliar with this rare zoonosis in humans [2,6]. Indeed, S. suis, a complex population consisting of heterogeneous strains, and a Gram-positive facultative anaerobe, can be classified into many serotypes based on the differentiation of capsule antigens $[7,8]$. Based on the varied virulence of these bacteria, they may be categorized into highly-pathogenic, weaklypathogenic, and nonpathogenic strains $[9,10]$. 
S. suis infection is acquired from pigs, either during slaughtering or by handling and eating undercooked pork products including abattoir workers, butchers, and pig breeders. S. suis should be considered as food-borne pathogen and it is potentially preventable $[3,11,12]$. These infections include meningitis, septicemia, pneumonia, endocarditis, arthritis, endophthalmitis, and septic shock. S. suis disease in humans is rare, probably underdiagnosed infection that usually occurs as sporadic cases. The treatment of $S$. suis infection requires several weeks of intravenous antimicrobial drugs $[4,7,13,14]$.

The outcome in survivors is marked by permanent hearing loss or vestibular dysfunction and deafness of perceptive type as commonly occurring sequelae. Death is exceptional and high mortality rates were observed especially in immunocompromised patients $[3,8,15]$. The objective of this work is to report a rare case of meningitis due to streptococcus suis.

\section{Case Presentation}

A 32-year-old young adult man was admitted for severe headaches and intense agitation. He is a pig farmer and a pork butcher. He was previously healthy man with no medical history. He was transferred from a district hospital for better management of vomiting, fever, and chills that began 72 hours ago. At the time of admission, he had a body temperature of $37,4{ }^{\circ} \mathrm{C}$, blood pressure of $110 / 70 \mathrm{mmhg}$, and a glycemia level of $1.8 \mathrm{~g} / \mathrm{l}$. The general state was good. The consciousness state was normal on Glasgow coma score scale. Regarding the neurological exam, there were no motor and sensitive deficits. Cerebellar and cranial nerves signs were not observed. Eyes movements were normal. The cardiovascular, respiratory and digestive exams were also normal. Acute meningeal hemorrhage was proposed as the initial diagnostic. Lumbar puncture was done, and the clear cerebrospinal fluid sample was sent to the lab for checking.

At the second day of admission, he felt cold with fever $39.5^{\circ} \mathrm{C}$, agitation, severe headaches and vomiting. The neck become stiff with positive Kernig and Brudzinski signs. The second CSF was turbid without blood. A diagnostic of bacterial meningitis was made according to the meningeal febrile syndrome. Empirical antibiotics were used in the waiting for the ADST.

Laboratory results had shown increased leukocytes count (2800/ $\mathrm{mm}^{3}$ with lymphocytes $65 \%$ and neutrophils $35 \%$ ), an increasead of protein concentration at $1.8 \mathrm{~g} / \mathrm{l}$, and glucose level of $0.47 \mathrm{~g} / \mathrm{l}$ and no organism on Gram stain. CSF culture grew small alpha-hemolytic colonies on sheep blood agar plates. The microorganisms which were Gram-positive cocci either in chains or in pair were catalase- negative. They were identified as Streptococcus suis serotype I by VITEK 2 gram-positive card system (BioMérieux), (Figure 1). The sample was sent to another lab in France which confirmed three weeks later the same serotype. Antimicrobial drug-susceptibility testing (ADST) performed in Vitek 2 and minimal inhibitor concentration (MIC) interpreted according to EURCAST revealed a strain sensitive to penicillin (MIC $\leq 0.25$ $\mathrm{mg} / \mathrm{l}$ ) levofloxacin, moxifloxacin, linezolide and nitrofurantoine and resistant to clindamycine and tetracycline.
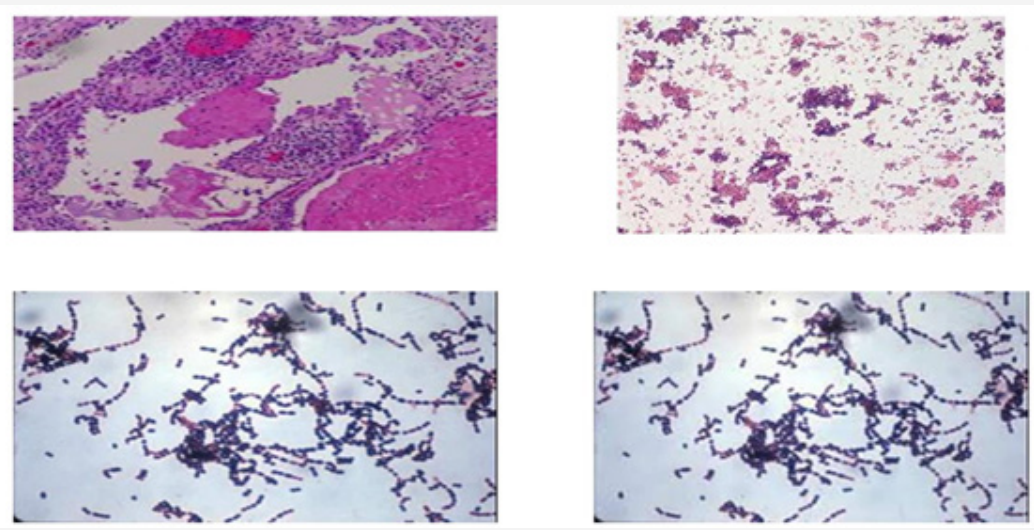

Figure 1: CSF and blood samples showed histological and histochemical aspects of Streptococcus Suis as small alpha-hemolytic colonies on sheep blood agar plates. The microorganisms were Gram-positive cocci either in chains or in pair were catalase- negative. They were identified as Streptococcus suis serotype I by VITEK 2 gram-positive card system (BioMérieux).
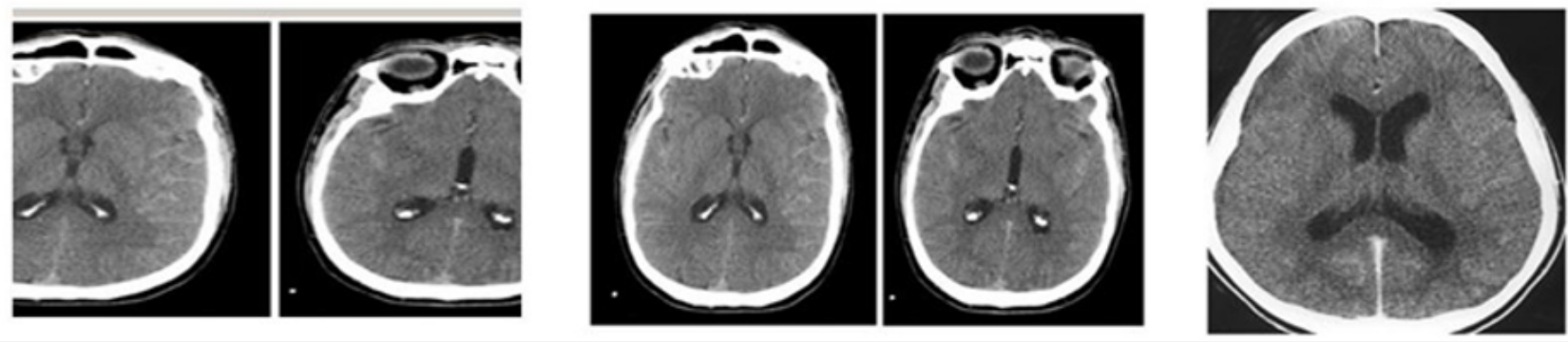

Figure 2: CT scan axial section with contrast showed enhancement of meningeal spaces. 

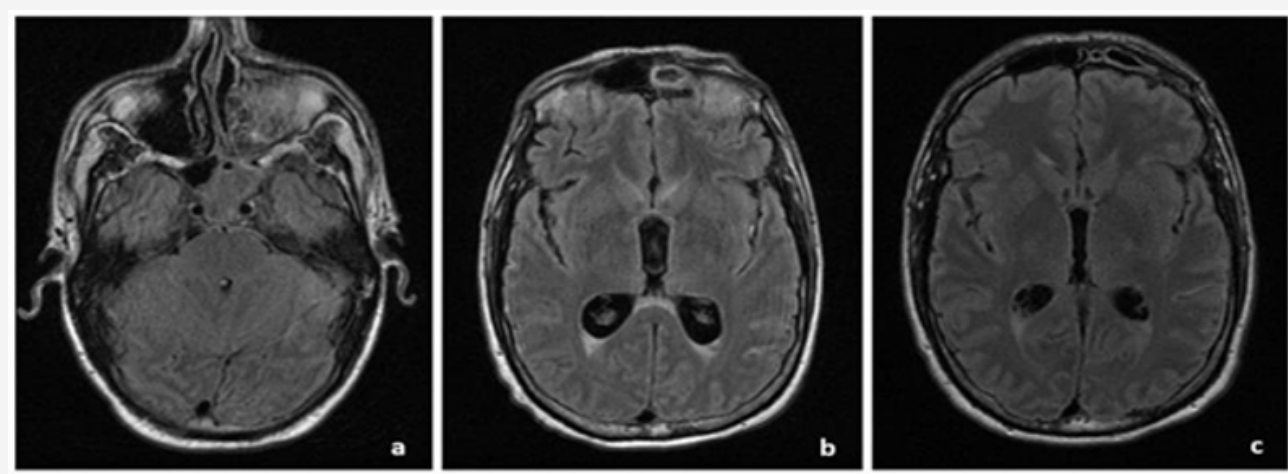

Figure 3: Brain MRI with axial and sagittal sections showed a T1 weighted hyposignal and T2-weighted hypersignal of meningeal spaces.

The CT scan and MRI showed enhancement of meningeal spaces, (Figure $2 \& 3$ ). After fifteen days of treatment the patient had improved better with continuous follow up to twenty-one days. All the meningeal symptoms were stopped, and the patient had good recovery. During regular control, the CSF was aseptic and clear with no microorganisms. After three months of follow up and rehabilitation, the patient showed perceptive hearing loss with balance disturbance as the sequels. Physicians must bear in mind that some psychiatric patients may be the serious infectious patients.

\section{Discussion}

Our data has several limitations. The study is a case report from a pigs beerer pork butcher. Bacterial meningitsi is frequent in our region especially in dry season and often due to Neisseria Meningococci. $S$ suis meningitis is rare and have never been reported, maybe due to no focus on related research. Then many of these meningitis types are underdiagnosed by the clinical and technical recruitment bias and this solely case cannot reflect the real frequence of the infection. Blood and CSF cultures were not necessarily performed for all patients with suggested meningitis, possibly resulting in missed cases or another biasing in our study toward the more clinically apparent or severe cases $[3,8,11]$.

S. suis meningitis infections are typically sporadic, and, in most cases, occur in occupational groups, such as abattoir workers and butchers. Infection may also be acquired by contact with raw or undercooked meat products $[10,12,15]$. Our patient had the both function as a pig farming and a pork butcher.

Due to high pork consumption and frequent small-scale pig rearing, $S$. suis infection is endemic worldwide but the most important cases were reported in The Netherlands and SouthEast- Asia [4,6,14]. However, most clinicians and microbiologists are unfamiliar with this rare zoonosis in humans. Also, our clinical laboratory platform equipment did not allow to perform the search of this subtype of bacteria. Meningitis is the most frequently described presentation of $S$. suis infection, occurring in approximately $50-60 \%$ of infected patients $[7,9,10,16]$.

S. suis is responsible for sporadic as well as epidemic infections in pigs. Healthy carrier pigs harbour the organism in their nose and palatine tonsils $[5,6]$. Although invasive illness occurs in a minority of colonized animals, these carriers play an important role in disseminating the infection $[4,5]$. The most frequent transmission route is through skin abrasions or cuts, although in some cases, no skin laceration, and any contact can be found $[3,12,13,17]$.

Here we report a clinical and laboratory-confirmed cases of $S$. suis meningitis. Our patient was admitted with symptoms and signs leading first to meningeal hemorrhage and secondly to bacterial meningitis. Because his several years of exposure due to his job as pig farming and pork butcher, $S$ suis was suspected and it is under taken the process to identify the serotype and the species. The CSF and blood samples were sent to laboratory in France. It was identified with confirmation three weeks later as $S$ suis serotype 1.

The bacteriological diagnosis of $S$ suis is not always easily established in our country in daily practice. $S$ suis grows on sheep or bovine blood agar as beta-, alpha- or nonhemolytic colonies. Alpha-hemolytic isolates can grow mucoid colonies like those of pneumococci. Because many laboratories are unaware of this organism, it can easily be mistaken for enterococci. Since the first case had been described up to today, many serotypes were established and lot of especies were identified worldwide $[4,6,11,18]$.

Therefore, S. suis meningitis patients with negative blood cultures might have been missed. Furthermore, because comprehensive examinations were not performed on patients after discharge, neurologic or cognitivie sequalae might have been also misdiagnosed in African countries [5,8,19].

The patient had received early administration of intravenous ceftriaxone and dexamethasone. The patient had complete recovery from meningeal syndrome but still with sequels like hearing loss perceptive type and slight balance problem. Some previous studies have reported these common complications of $S$ suis meningitis with an incidence rate of $54 \%$ to $64 \%$. [2,15,20] The outcome was favourable without heavy sequels and death in our reported case, but some authors have observed $7 \%$ of mortality rate. However, eradication of the organism can be difficult, and some cases of relapse after four weeks of treatment have been reported. Therefore, some authors suggest six to eight-week treatment for every case of $S$ suis meningitis [12,21-23].

\section{Conclusion}

Here we have reported a clinical and laboratory-confirmed first case of uncommon $S$. suis infection in our country. Patient 
was treated promptly and early to prevent cerebral and cognitive sequelae. In low income countries most hospital laboratories are not able to confirm $S$. suis, the infection might be misdiagnosed. Clinicians in high-risk areas of bacterial meningitis must keep this in mind and be aware of this subtype of species. Active surveillance is needed in our region to put in action all preventives measures among pig farming and pork butchers.

\section{Acknowledgemnet}

None.

\section{Conflict of Interest}

No conflict of interest.

\section{References}

1. Thi Lan HV, Ngo Ha, Nguyen TH, Horby Peter, Dang Trung NH, et al. (2014) Epidemiology, Clinical Manifestations, and Outcomes of Streptococcus suis Infection in Humans. Emerging Infectious Diseases 20(7): 1105-1114.

2. Choi SM, Cho BH, Choi KH, Nam TS, Kim JT, et al. (2012) Meningitis Caused by Streptococcus suis: Case Report and Review of the Literature. J Clin Neurol 8(1): 79-82.

3. Praphasiri P, Owusu JT, Thammathitiwat S, Ditsungnoen D, Boonmongkon P, et al. (2015) Streptococcus suis Infection in Hospitalized Patients, Nakhon Phanom Province, Thailand. Emerging Infectious Diseases 21(2): 345-348.

4. Edmond K, Clark A, Korczak VS, Sanderson C, Griffiths UK, et al. (2010) Global and regional risk of disabling sequelae from bacterial meningitis: a systematic review and meta-analysis. Lancet Infect Dis 10: 317-328.

5. Fongcom A, Pruksakorn S, Netsirisawan P, Pongprasert R, Onsibud P (2009) Streptococcus suis infection: a prospective study in northern Thailand. Southeast Asian J Trop Med Public Health 40(3): 511-517.

6. Ferrando ML, de Greeff A, van Rooijen WJ, Stockhofe-Zurwieden N Nielsen J, et al. (2015) Host-pathogen interaction at the intestinal mucosa correlates with zoonotic potential of Streptococcus suis. J Infect Dis 212(1): 95-98.

7. Tang J, Wang C, Feng Y, Yang W, Song H, et al. (2006) Streptococcal toxic shock syndrome caused by Streptococcus suis serotype 2. PLoS Med 3(5): e151-155.

8. Navacharoen N, Chantharochavong V, Hanprasertpong C, Kangsanarak J, Lekagul S (2009) Hearing and vestibular loss in Streptococcus suis infection from swine and traditional raw pork exposure in northern Thailand. J Laryngol Otol 123(8): 857-862.

9. Rusmeechan S, Sribusara P (2008) Streptococcus suis meningitis: the newest serious infectious disease. J Med Assoc Thai 91(5): 654-658.
10. van Samkar A, Brouwer MC, Schultsz C, van der Ende A, van de Beek D (2015) Streptococcus suis Meningitis: A Systematic Review and Metaanalysis. PLoS Negl Trop Dis 9(10): e0004191.

11. Kerdsin A, Akeda Y, Hatrongjit R, Detchawna U, Sekizaki T, et al. (2014) Streptococcus suis serotyping by a new multiplex PCR. J Med Microbiol 63(Pt6): 824-830.

12. Bojarska A, Molska E, Janas K, Skoczyńska A, Stefaniuk E, et al. (2016) Streptococcus suis in invasive human infections in Poland: clonality and determinants of virulence and antimicrobial resistance. Eur J Clin Microbiol Infect Dis 35(6): 917-925.

13. Goyette-Desjardins G, Auger J-P, Xu J, Segura M, Gottschalk M (2014) Streptococcus suis, an important pig pathogen and emerging zoonotic agent-an update on the worldwide distribution based on serotyping and sequence typing. Emerg Microbes Infect 3(6): e45-46.

14. Nga TV, Nghia HD, Tu le TP, Diep TS, Mai NT, et al. (2011) Real-time PCR for detection of Streptococcus suis serotype 2 in cerebrospinal fluid of human patients with meningitis. Diagn Microbiol Infect Dis 70(4): 461467.

15. Trung NHD, Phuong TLH, Wolbers M, Minh HNV, Nguyen Thanh V, et al. (2012) Aetiologies of central nervous system infection in Viet Nam: a prospective provincial hospital-based descriptive surveillance study. PLoS One 7: e378258-378261.

16. Zaccaria E, van Baarlen P, de Greeff A, Morrison DA, Smith H, et al. (2014) Control of competence for DNA transformation in Streptococcus suis by genetically transferable pherotypes. PLoS One 9(6): e99394.

17. Manzin A, Palmieri C, Serra C, Saddi B, Princivalli MS, et al. (2008) Streptococcus suis Meningitis without History of Animal Contact, Italy. Emerging Infectious Diseases 14(12): 1946-1948.

18. Haas B, Bonifait L, Vaillancourt K, Charette SJ, Gottschalk M, et al. (2014) Characterization of DNase activity and gene in Streptococcus suis and evidence for a role as virulence factor. BMC Res Notes 7: 424-429.

19. Zheng JX, Li Y, Zhang H, Fan HJ, Lu CP (2013) Identification and characterization of a novel hemolysis-related gene in Streptococcus suis serotype 2. PLoS One 8(9): e74674.

20. Zhao Y, Liu G, Li S, Wang M, Song J, et al. (2011) Role of a type IVlike secretion system of Streptococcus suis 2 in the development of streptococcal toxic shock syndrome. J Infect Dis 204(2): 274-281.

21. Gottschal KM, Xu J, Lecours M-P, Grenier D, Fittipaldi N, et al. (2010) Streptococcus suis infections in humans: what is the prognosis for Western countries? (Part II). Clin Microbiol News l 32(1): 97-102.

22. Feng Y, Zhang H, Ma Y, Gao GF (2010) Uncovering newly emerging variants of Streptococcus suis, an important zoonotic agent. Trends Microbiol 18(3): 124-131.

23. Zalas-Wiecek P, Michalska A, Grabczewska E, Olczak A, Pawlowska M, et al. (2013) Human meningitis caused by Streptococcus suis. J Med Microbiol 62(Pt 3): 483-485. 